\title{
Cognition in pregnancy and motherhood: prospective cohort study
}

\author{
Helen Christensen, Liana S. Leach and Andrew Mackinnon
}

\section{Background}

Research has reported that pregnant women and mothers become forgetful. However, in these studies, women are not recruited prior to pregnancy, samples are not representative and studies are underpowered.

\section{Aims}

The current study sought to determine whether pregnancy and motherhood are associated with brief or long-term cognitive deterioration using a representative sample and measuring cognition during and before the onset of pregnancy and motherhood.

\section{Method}

Women aged 20-24 years were recruited prospectively and assessed in 1999, 2003 and 2007. Seventy-six women were pregnant at follow-up assessments, 188 became mothers between study waves and 542 remained nulliparous.

\section{Results}

No significant differences in cognitive change were found as a function of pregnancy or motherhood, although late pregnancy was associated with deterioration on one of four tests of memory and cognition.

\section{Conclusions}

The hypothesis that pregnancy and motherhood are associated with persistent cognitive deterioration was not supported. Previous negative findings may be a result of biased sampling.

\section{Declaration of interest}

None.
Many pregnancy guidebooks, the popular press and websites counsel pregnant women on the possibility of short-term memory problems during pregnancy, describing the condition as 'baby brain' or 'placenta brain. ${ }^{1-3}$ These views are supported by scientific research evidence and systematic reviews. ${ }^{4-20}$ For example, a recent systematic review found that pregnant women perform more poorly than non-pregnant women on memory and other cognitive tests. ${ }^{4}$ Similar deficits are reported in motherhood in crosssectional ${ }^{19}$ and longitudinal studies ${ }^{13,18}$ (but see Crawley et al). ${ }^{17}$ However, the animal-model literature paints a radically different picture of the effects of pregnancy and motherhood on cognitive capabilities. Kinsley et $a l^{21}$ reported better spatial learning and memory during pregnancy in rats, as have others. ${ }^{22}$ These improvements persist. In motherhood, parous rats compared with nulliparous rats navigated mazes more efficiently, ${ }^{23}$ experienced less anxiety and fear, as indexed by levels of stress hormones in the blood and by behaviour in open fields. ${ }^{24,25}$ Although the tests used to measure cognitive functioning in rodents and humans differ markedly, it is surprising that outcomes of the human and rat research appear to be at such odds, given that 'most mammals share similar maternal behaviours, which are probably controlled by the same brain regions in both humans and rats. ${ }^{25}$ This suggested to us that the effect of pregnancy or motherhood on cognitive abilities may not have been adequately tested. No study of human mothers has collected or examined data on cognitive performance prior to pregnancy, the samples recruited to the studies were effectively convenience samples, with many women recruited as volunteers from prenatal classes and matched to non-pregnant 'friends' and, although studies examined for the possibility of brief impairments immediately pre- and post-birth, few studies examined long-term effects, months or years after pregnancy or motherhood. The current study sought to determine whether pregnancy and motherhood are associated with cognitive deterioration using a study that measured baseline cognitive performance before pregnancy and motherhood, and used a large representative sample. Hypotheses were framed in terms of deficits. To test our first hypothesis (hypothesis one) that pregnancy leads to impaired cognitive functioning, we compared women pregnant at wave two with women not pregnant at or before wave two on change in cognitive function from wave one to wave two. Analogously, change from wave two to wave three was compared between those pregnant at wave three and those not pregnant at or before wave three. To assess short-term effects we examined the effects of length of pregnancy on cognitive performance as a function of stage of pregnancy (1-4 months $v$. 5 months or more). To investigate whether the birth of a child (i.e. motherhood) impaired cognitive capacity (hypothesis two), we compared women who became pregnant between waves one and two and who were, therefore, new mothers at wave two with women not pregnant at or before wave two. Similarly, we compared women who became pregnant between waves two and three and who were new mothers at wave three with women not pregnant at or before wave three. Four areas of cognition were assessed. These were cognitive speed, working memory, immediate and delayed recall.

\section{Method}

\section{Participants}

The Personality and Total Health (PATH) Through Life Project is a prospective longitudinal narrow-age cohort community survey concerned with health and well-being. Three cohorts were recruited in 1999 (20-24 years, 40-44 years and 60-64 years). ${ }^{26}$ The sample for this study consisted of women from the youngest cohort. In this cohort, 2404 individuals aged 20-24 years were recruited from the electoral roll with a recruitment rate of $58.6 \%$. Of these, 1241 were women who completed the baseline measurement in 1999. Subsequently, 1126 and 1058 women completed the first and second follow-up in 2003 and 2007. This represented a follow-up rate of $91 \%$ and $85 \%$ respectively. Of the 115 participants who were not interviewed beyond the first wave, 83 refused or were unable to be interviewed for medical reasons, 
30 could not be located and 2 died between the waves. Only women who had completed at least both waves one and two were considered in the present analyses. Ethics approval was obtained from the Australian National University's Human Research Ethics Committee.

Over the 8 years of the study, 76 were pregnant at follow-up (2003 or 2007) and 188 women became mothers (but were not pregnant at the time of the interview). Only primigravidae and first-time mothers were studied because multiple pregnancies and mothering of more than one child would introduce additional factors. Individuals becoming pregnant or becoming mothers between 1999 and 2003 were considered separately to women becoming pregnant or mothers between 2003 and 2007. In the analyses the two waves were compared separately because, over the 8-year period of the study, well-documented changes in IQ, as demonstrated in standard IQ scales such as Wechsler Adult Intelligence Scale, ${ }^{27}$ would be expected as a result of maturation, practice effects and additional education. Further, participants who did not become pregnant or progress to motherhood at any time point may have had characteristics that were different from those not yet pregnant. The PATH survey has a broad focus and provided no cue that cognitive performance with respect to either pregnancy or motherhood was a focus of attention.

\section{Survey procedure}

Individuals selected at random from the electoral roll were sent a letter informing them of the survey and that an interviewer would contact them soon to see if they wanted to participate. If a person agreed to participate, the interviewer arranged to meet them at some convenient location, usually the participant's home or the Centre for Mental Health Research at the Australian National University. Most of the interview was self-completed on a palmtop or laptop computer. However, testing by the interviewer was required for the physical tests and for some of the cognitive tests.

\section{Cognitive tests}

Four domains of cognitive functioning were assessed using tests that are sensitive to change: cognitive speed, working memory, immediate recall and delayed recall.

\section{Cognitive speed}

Mental speed was measured with the Symbol-Digit Modalities Test (SDMT), which asks the participant to substitute as many digits for symbols as possible in $90 \mathrm{~s}^{28}$

\section{Working memory}

Working memory was assessed with the Digits Backwards subtest of the Wechsler Memory Scale, ${ }^{29}$ which presents participants with series of digits at the rate of one per second and asks them to repeat the digits backwards.

\section{Immediate and delayed recall}

These were assessed with the first trial of the California Verbal Learning Test, ${ }^{30}$ which involves recalling a list of 16 nouns. The interval between immediate and delayed recall was occupied by a test of grip strength.

Cognitive change scores were calculated for each test by subtracting wave one scores from wave two scores.

\section{Predictor or control variables}

Education

Educational attainment was measured using six questions concerning the full spectrum of past and current primary (elementary), secondary and tertiary educational attainment. Responses to these questions were coded into a single measure corresponding to the number of years of education. For the purposes of the analyses, education was then categorised into four groups: 0-12 years, 13 years (i.e. high school), 14-15 years and 16 years or more.

\section{Depression}

Depression was measured by the Goldberg Depression Scale. ${ }^{31}$ The Goldberg Depression Scale consists of nine items, which are rated with a 'yes/no' response. Total scale scores are calculated by summing the number of 'yes' responses.

Anxiety

Anxiety was measured by the Goldberg Anxiety Scale. ${ }^{31}$ The Goldberg Anxiety Scale consists of nine items, which are rated with a 'yes/no' response. Total scale scores are calculated by summing the number of 'yes' responses.

\section{Other}

Antidepressant medication or anxiolytic medication was assessed by asking whether participants were 'taking any depression medication' (yes/no), or 'taking anxiety medication' (yes/no). Participants were also asked their marital status (married/de facto married, not married), and whether they had sleeping difficulties, using one item 'sleeping poorly' (yes/no).

\section{Cohorts}

We sought to maximise available data at each wave and to use appropriate groups for comparison. Change in those pregnant at waves two and three were compared with change in contemporaneously non-pregnant women in separate contrasts. Seven relevant patterns of pregnancy and motherhood were identified and subgroups formed on the basis of these (Fig. 1). Groups one and two consisted of women who were either pregnant at the first wave of the study or who had been so beforehand. Because no information was available about the cognitive status of these women before they became pregnant or mothers these women could not be included in the study. Groups three to six comprise women who became pregnant at different stages of the study. Group seven are women who had never been pregnant up until the last occasion of measurement. The groups were variously used to test hypotheses as outlined in Fig. 1.

\section{Statistical analyses}

One-way analyses of variance (ANOVAs) were used to test baseline differences between each of the relevant subgroups for each of the cognitive variables. Mixed model repeated measures ANOVA ${ }^{32}$ with group (as defined in Fig. 1) and wave as factors were fitted to each variable. Within-participant variation was modelled using an unstructured covariance matrix. The outcomes of conventional tests of each main effect and the interaction were not relevant to this study. Testing each hypothesis involved specifying a two degree of freedom contrast composed of the comparison appropriate subgroups for wave one to wave two change and an analogous comparison for wave two to wave three change. Results from each comparison were also available, enabling exploration of effects present only for wave two or for wave three. The numbers of participants with missing data for each comparison are given in the footnote to the corresponding tables. Sensitivity analyses indicated that, given the size of relevant subgroups, individual contrasts would have $80 \%$ power to detect between-group differences in the range 0.3 to 0.5 standard deviations.

To examine the effects of pregnancy and recency of motherhood on cognitive functioning we categorised pregnant women 


\begin{tabular}{|c|c|c|c|c|c|c|c|c|c|c|c|}
\hline Group & Pre-wave 1 & Wave 1 & Interwave 1-2 & Wave 2 & Interwave 2-3 & Wave 3 & $\mathrm{H}_{1}$ & $\mathrm{H}_{1}$ & $\mathrm{H}_{2}$ & $\mathrm{H}_{2}$ & \\
\hline & & & & & & & W1-W2 & W2-W3 & W1-W2 & w2-w3 & $n$ \\
\hline 1 & M & M & $M / P$ & $M / P$ & M/P/DO & $\mathrm{M} / \mathrm{P} / \mathrm{DO}$ & & & & & 151 \\
\hline 2 & $\mathrm{~N}$ & P & $M / P$ & $M / P$ & M/P/DO & $\mathrm{M} / \mathrm{P} / \mathrm{DO}$ & & & & & 20 \\
\hline 3 & $\mathrm{~N}$ & N & P & M & $\mathrm{M} / \mathrm{P} / \mathrm{DO}$ & $\mathrm{M} / \mathrm{P} / \mathrm{DO}$ & & & M & & 76 \\
\hline 4 & $\mathrm{~N}$ & $\mathrm{~N}$ & $\mathrm{~N}$ & P & $\mathrm{M} / \mathrm{P} / \mathrm{DO}$ & $\mathrm{M} / \mathrm{P} / \mathrm{DO}$ & P & & & & 30 \\
\hline 5 & N & $\mathrm{N}$ & N & $\mathrm{N}$ & P & & $\bar{P}$ & & $\bar{M}$ & M & 112 \\
\hline 6 & N & $\mathrm{N}$ & $\mathrm{N}$ & $\mathrm{N}$ & $\mathrm{N}$ & $\bar{P}$ & $\overline{\mathrm{P}}$ & P & $\bar{M}$ & & 46 \\
\hline 7 & $\mathrm{~N}$ & N & $\mathrm{N}$ & N & N/DO & N/DO & $\bar{P}$ & $\bar{P}$ & $\bar{M}$ & $\overline{\mathrm{M}}$ & 542 \\
\hline
\end{tabular}

\section{Fig. 1 Cohorts defined by pregnancy status at each wave and interwave interval.}

N, Non-mother; P, Pregnant; M, Mother; DO, participant who dropped out.

Group 1: Mothers at entry; group 2: pregnant wave 1; group 3: new mothers wave 2; group 4: pregnant wave 2; group 5: new mothers wave 3; group 6: new mothers wave 3; group 7: never pregnant. Additional groups not shown in the figure: 115 women who dropped out post-wave 1 and 149 women who experienced multiple pregnancies between waves of data collection.

$\mathrm{H}_{1}$ refers to hypothesis one that pregnancy is associated with impaired functioning. Two contrasts compared women pregnant at the time of assessment (P) with those women not (yet) pregnant ( $\bar{P})$. The first contrast (W2-W1) involved women pregnant at wave 2, the second (W3-W2), women pregnant at wave 3. $\mathrm{H}_{2}$ refers to hypothesis two that motherhood leads to impaired cognitive functioning. Two contrasts compared women who had become mothers between a previous and the next assessment (M) with women who were not (yet) mothers $(\bar{M})$. The first contrast (W2-W1) involves women who became mothers at wave 2, the second (W3-W2), women who became mothers at wave 3.

into early and late pregnancy. To examine the effects of recent transition to motherhood, we divided women into those with babies under 12 months at the time of testing and those with older infants. Choice of categorisations was moderated by sample size considerations. Significance was set at $P=0.05$.

\section{Results}

One-way analyses of variance (ANOVAs) did not reveal baseline differences between the relevant sample groups on the tests of cognitive function (Tables 1 and 2). To examine the effects of potential confounding variables, the relationship of each cognitive outcome with age, education, depression, anxiety, taking medication for anxiety or depression, marital status and 'sleeping poorly' was investigated (time-varying for each occasion of measurement). Only education was identified as a significant covariate for all of the cognitive factors (all $P<0.001$ ). However, further investigations comparing the adjusted and unadjusted models and estimated means for the outcome variables clearly demonstrated that the effect of education was negligible. Therefore, the analyses reported below were not adjusted for education (or any of the other potential covariates).

The pre-specified contrasts within mixed model repeated measures ANOVAs found no significant differences in cognitive change from waves one to two between those who were pregnant and those who were not on tests of cognitive speed (SDMT) and memory recall (immediate recall, delayed recall) (Fig. 2 and Table 3). There was one significant effect in favour of non-pregnant women on working memory (Digits Backwards subtest) between waves two and three. Those who became pregnant at wave three experienced a greater decline in their Digits Backwards score than those who remained non-pregnant (mean difference in change scores $-0.640, P=0.037)$. However, the aggregate effect over the

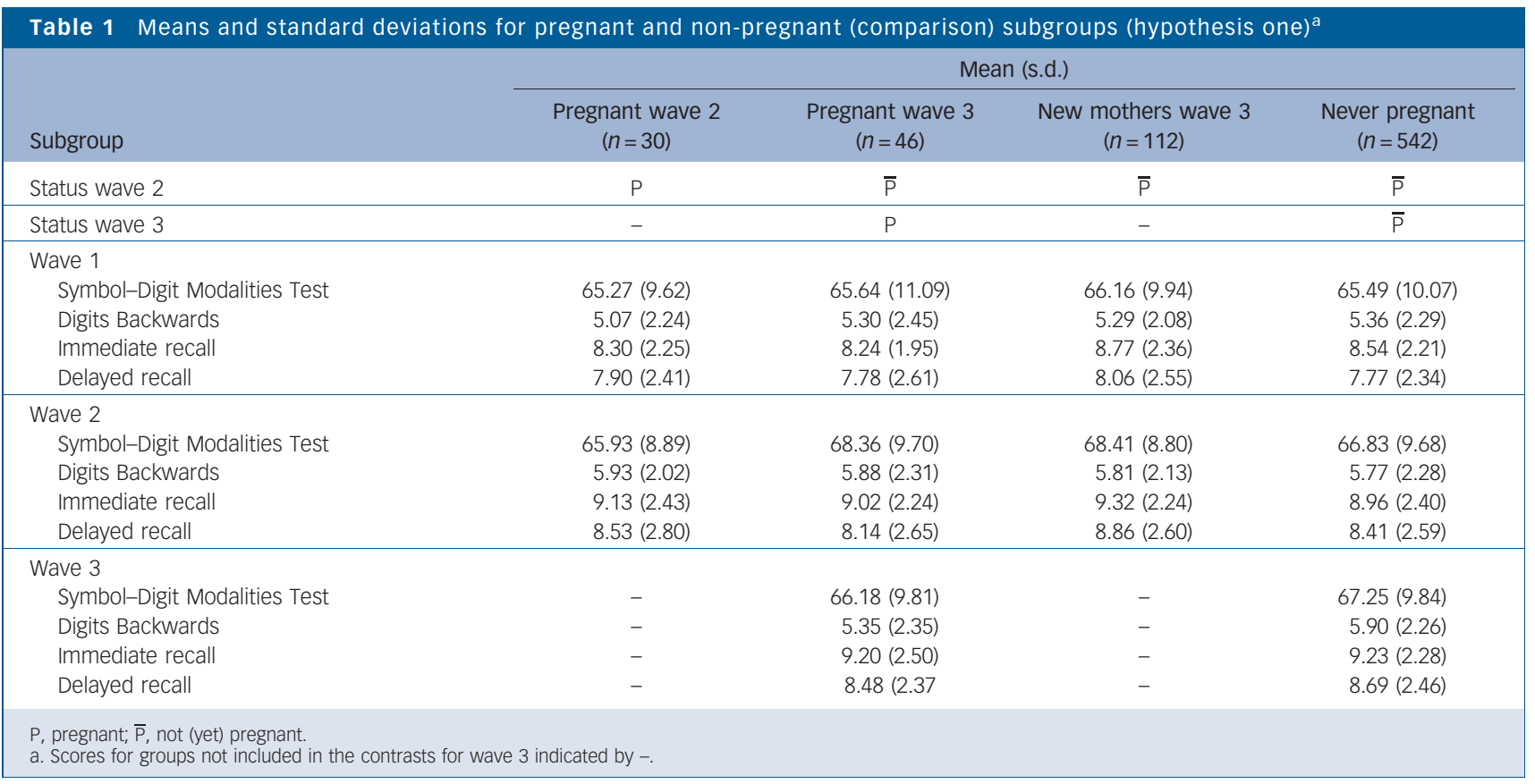




\begin{tabular}{|c|c|c|c|c|}
\hline \multirow[b]{2}{*}{ Subgroup } & \multicolumn{4}{|c|}{ Mean (s.d.) } \\
\hline & $\begin{array}{c}\text { New mothers wave } 2 \\
(n=76)\end{array}$ & $\begin{array}{l}\text { New mothers wave } 3 \\
\qquad(n=112)\end{array}$ & $\begin{array}{l}\text { Pregnant wave } 3 \\
(n=46)\end{array}$ & $\begin{array}{l}\text { Never pregnant } \\
\quad(n=542)\end{array}$ \\
\hline Status wave 2 & M & $\bar{M}$ & $\bar{M}$ & $\bar{M}$ \\
\hline Status wave 3 & - & M & - & $\overline{\mathrm{M}}$ \\
\hline \multicolumn{5}{|l|}{ Wave 1} \\
\hline Symbol-Digit Modalities Test & $64.60(9.14)$ & $66.16(9.94)$ & 65.64 (11.09) & 65.49 (10.07) \\
\hline Digits Backwards & $4.78(2.36)$ & $5.29(2.08)$ & $5.30(2.45)$ & $5.36(2.29)$ \\
\hline Immediate recall & $8.28(2.17)$ & $8.77(2.36)$ & $8.24(1.95)$ & $8.54(2.21)$ \\
\hline Delayed recall & $7.67(2.31)$ & $8.06(2.55)$ & $7.78(2.61)$ & $7.77(2.34)$ \\
\hline \multicolumn{5}{|l|}{ Wave 2} \\
\hline Symbol-Digit Modalities Test & $65.67(8.98)$ & $68.41(8.80)$ & $68.36(9.70)$ & $66.83(9.68)$ \\
\hline Digits Backwards & $5.14(2.07)$ & $5.81(2.13)$ & $5.88(2.31)$ & $5.77(2.28)$ \\
\hline Immediate recall & $8.34(2.13)$ & $9.32(2.24)$ & $9.02(2.24)$ & $8.96(2.40)$ \\
\hline Delayed recall & $7.84(2.48)$ & $8.86(2.60)$ & $8.14(2.65)$ & $8.41(2.59)$ \\
\hline \multicolumn{5}{|l|}{ Wave 3} \\
\hline Symbol-Digit Modalities Test & - & $66.89(8.64)$ & - & $67.25(9.84)$ \\
\hline Digits Backwards & - & $6.13(2.36)$ & - & $5.90(2.26)$ \\
\hline Immediate recall & - & $9.61(2.45)$ & - & $9.23(2.28)$ \\
\hline Delayed recall & - & $9.07(2.76)$ & - & $8.69(2.46)$ \\
\hline
\end{tabular}

two waves was not significant and if correction had been made for multiple testing, the effect would not be significant.

There were no significant differences in cognitive change between waves one and two for those who had become mothers between waves and those who had not. This was also the case for cognitive change between waves two and three (Fig. 3 and Table 4). The mean cognitive scores for each sample group across time can be seen in Fig. 3. Performance improved in all groups.

Secondary analyses examining stage of pregnancy and time since birth were also undertaken. Women pregnant at wave two and at wave three were divided into those only recently pregnant (5 or more months remaining) and those in later stages (last 4 months of pregnancy). Women in each of these groups were compared separately with the non-pregnant groups on each measure. The only effect found was on the SDMT for women in the later stages of pregnancy. Performance on this test fell from the previous wave by 2.60 points and 2.79 points for women pregnant at waves two and three respectively, whereas non-pregnant women recorded an improvement $(2.23$ points at wave two $)$ or stability ( 0.13 point drop at wave three). These contrasts were significant $\left(t_{687.7}=2.000, \quad P=0.046 \quad\right.$ and $\left.t_{646.5}=2.138, P=0.033\right)$. There were no effects for working memory or immediate or delayed recall. Secondary analysis of the motherhood data compared recent (infant less than 12 months) with established motherhood (older). There were no significant differences, and numbers precluded additional analysis of women immediately post-birth.

\section{Discussion}

\section{Findings from the study}

In short, no negative cognitive impacts of pregnancy and motherhood were observed in the full sample in this representative prospective study, except for the Digits Backwards subtest at wave three. Pregnant women at wave three, but not wave two, remembered approximately one digit backwards fewer than prior to pregnancy. However, this isolated finding would not remain significant under adjustment for multiple testing and was not significant when both waves were combined. These results provided no support for the study's hypotheses with respect to long-term cognitive change as a function of pregnancy or motherhood. In secondary analyses, we did find that women in later pregnancy in both waves were poorer on the SDMT but not on any tests of memory functioning.

\section{Reasons for the discrepancy with extant literature}

Although we found an isolated effect of late pregnancy on speed of cognitive performance in women in late pregnancy, findings from this prospective study are not consistent with the bulk of the literature, which reports various forms of cognitive deficit in both pregnancy and motherhood. One interpretation of the inconsistency is that the findings from previous studies are biased. This could be because of the recruitment of volunteer mothers who may differ in significant ways from 'average' pregnant women, being more concerned or anxious about the effect of pregnancy on their cognitive status or more depressed or sleep deprived. Alternatively, control volunteers may have differed from the pregnant groups in ways other than their non-gravid status. They may have a greater investment in cognitive performance than the pregnant women. Previously reported effects have generally been modest and could well have resulted from relatively subtle biases to which samples of convenience and non-randomly assigned groups are at risk.

A second explanation is that more cognitive deficits do exist, but that the tests used failed to detect them. This is unlikely as the measures employed were the same or similar to those previously used with reported effects for working memory $y^{7,8,12}$ and speed. ${ }^{14,16}$ It remains possible that cognitive tests that reflect fluctuations in attention might reveal more subtle differences. Recently, Rendell and Henry have argued that pregnant women exhibited increased difficulties in implementing delayed intentions in daily life, and that these deficits emerged only outside of laboratory environments. ${ }^{8}$ Moreover, tests in the present study, like the majority of previous research, are not designed to detect whether any potential deficits were because of motivational or dispositional differences between pregnant and non-pregnant women. Some commentators have suggested that pregnant women may be less disposed to undertake cognitive testing, may regard it 

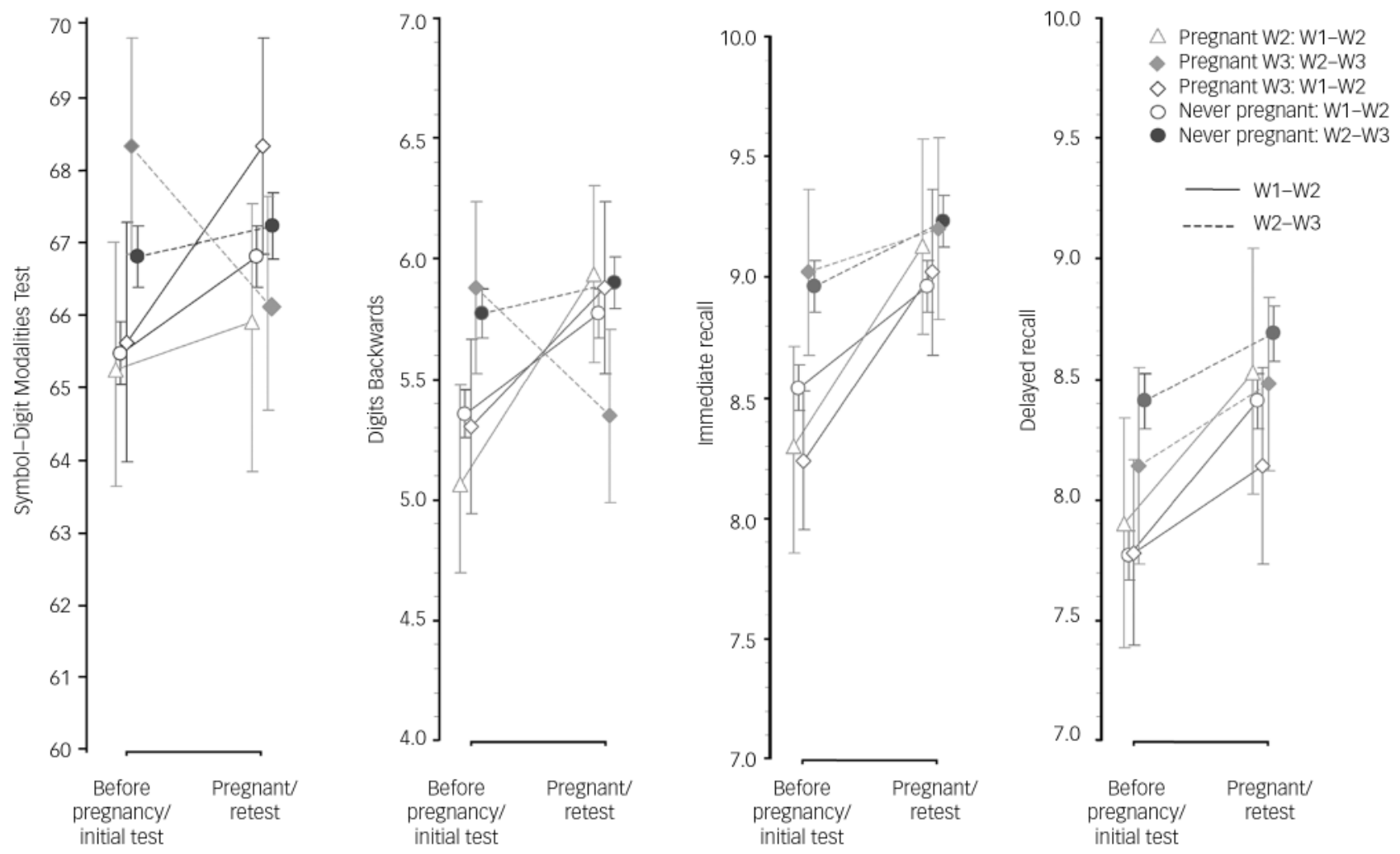

Fig. 2 Scores on cognitive tests as a function of pregnancy status.

Table 3 Mean differences in change scores for cognitive tests by pregnancy status for each wave

\begin{tabular}{|c|c|c|c|c|c|}
\hline \multirow[b]{2}{*}{ Test $^{\mathrm{a}}$} & \multicolumn{2}{|c|}{ Wave $1-2$} & \multicolumn{2}{|c|}{ Wave $2-3$} & \multirow{2}{*}{$\begin{array}{c}\text { Overall } \\
P\end{array}$} \\
\hline & Contrast value $^{\mathrm{b}}(95 \% \mathrm{Cl})$ & $P$ & Contrast value $^{\mathrm{b}}(95 \% \mathrm{Cl})$ & $P$ & \\
\hline Symbol-Digit Modalities Test & $-1.41(-4.22$ to 1.41$)$ & 0.327 & $-1.73(-3.70$ to 2.33$)$ & 0.084 & 0.160 \\
\hline Digits Backwards & $0.35(-0.41$ to 1.12$)$ & 0.365 & $-0.64(-1.25$ to -0.04$)$ & 0.037 & 0.058 \\
\hline Immediate recall & $0.24(-0.68$ to 1.15$)$ & 0.610 & $-0.07(-0.83$ to 0.68$)$ & 0.848 & 0.850 \\
\hline Delayed recall & $0.05(-0.87$ to 0.99$)$ & 0.914 & $0.13(-0.66$ to 0.92$)$ & 0.746 & 0.947 \\
\hline
\end{tabular}

as less important than control women, and thus perform more poorly because of poor motivation rather than diminished capacity. ${ }^{11}$ A limitation of this epidemiological study is that the tests in the current battery did not assess these specific, narrow abilities.

\section{Limitations of our study}

In interpreting the results the time between testing occasions needs to be considered. For the pregnant women, the prepregnancy assessments were up to 4 years before the testing during pregnancy. Women were at various stages of pregnancy at the time of testing. For these reasons, it is important to distinguish between short-term and longer-term effects. With the exception of one effect at wave two, our primary analyses indicated that there were no longer-term average effects of pregnancy on cognitive performance if all stages of pregnancy were combined. Our secondary analyses, which separated groups into those early and late in pregnancy, did find an effect for women late in pregnancy on a speed task within both waves, but this deficit was not found on three tests of memory. This finding underscores the importance of examining effects as a function of stage of pregnancy. Achieving this in unselected studies will be difficult because of the small numbers of women at each stage of pregnancy in even the largest studies. Regardless of this, the specific finding of a speed effect in late pregnancy requires replication.

For tests involving new mothers, the interval between not being a mother and becoming one was variable. For instance, some women had been mothers for periods of days whereas others had been mothers for years. There is no clear indication from the research literature as to when deficits might be likely to arise or to peak. Some studies have found memory deficits 32 weeks postpartum. ${ }^{13}$ Other studies have reported that the cognitive deficits are no longer apparent after short intervals of days. ${ }^{12}$ We found no correlation between the age of the child (i.e. period of motherhood) and cognitive deficits in supplementary analyses.

This study is the largest of its kind. Nevertheless it is limited in its ability to detect small effects because of the size of the pregnant and new mother groups. Steps were taken to maximise power and we did detect significant change in cognition (largely improvements) over the period. We were able to assess and exclude or 

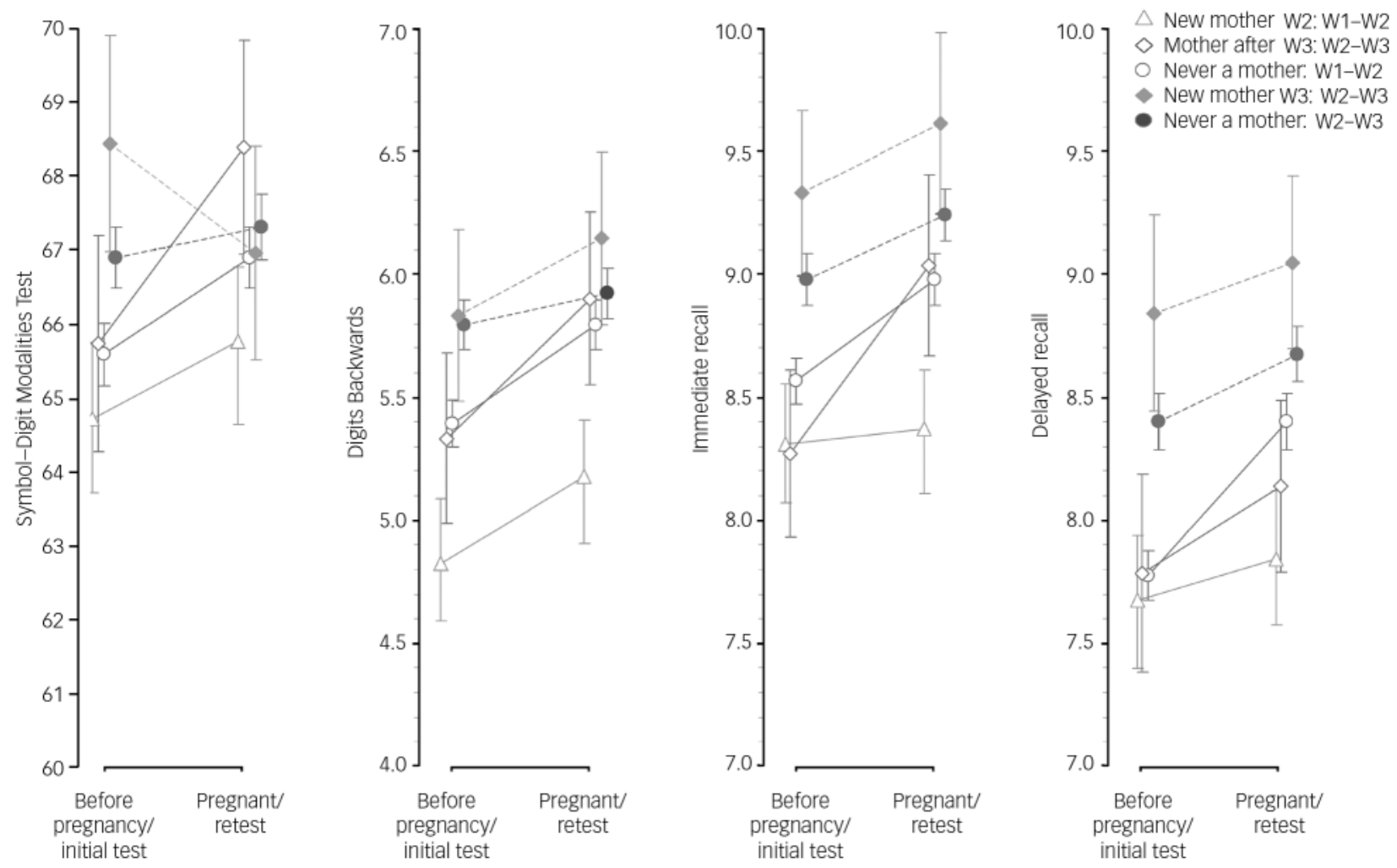

Fig. 3 Scores on cognitive tests as a function of motherhood status.

\begin{tabular}{|c|c|c|c|c|c|}
\hline \multirow[b]{2}{*}{ Test $^{\mathrm{a}}$} & \multicolumn{2}{|c|}{ Wave $1-2$} & \multicolumn{2}{|c|}{ Wave $2-3$} & \multirow{2}{*}{$\begin{array}{c}\text { Overall } \\
P\end{array}$} \\
\hline & $\begin{array}{l}\text { Contrast value } \\
\qquad(95 \% \mathrm{Cl})\end{array}$ & $P$ & $\begin{array}{l}\text { Contrast value }^{\mathrm{b}} \\
\qquad(95 \% \mathrm{Cl})\end{array}$ & $P$ & \\
\hline Symbol-Digit Modalities Test & $-1.02(-2.92$ to 0.87$)$ & 0.289 & $-1.21(-2.50$ to 0.09$)$ & 0.068 & 0.121 \\
\hline Digits Backwards & $-0.14(-0.66$ to 0.37$)$ & 0.583 & $0.24(-0.16$ to 0.64$)$ & 0.235 & 0.400 \\
\hline Immediate recall & $-0.53(-1.14$ to 0.08$)$ & 0.088 & $0.04(-0.46$ to 0.54$)$ & 0.878 & 0.223 \\
\hline Delayed recall & $-0.41(-1.04$ to 0.22$)$ & 0.198 & $-0.04(-0.56$ to 0.48$)$ & 0.889 & 0.437 \\
\hline
\end{tabular}

control the effects of depression, anxiety, medication, sleep problems, age and education on the test performance. In the absence of the possibility of mimicking animal studies and randomly assigning women to pregnancy or non-pregnancy conditions, the design of the present trial is as robust as possible in the context of human experimentation.

Finally, one of the weaknesses of our study was our inability to link cognitive change with biological changes associated with pregnancy. We were unable to look at the effects of contraceptive use in mothers and non-mothers. These analyses require targeted rather than epidemiological investigation. Our study, however, had the advantage of being a large population-based sample with pre-test measures, features that are not present in studies with retrospective recruitment.

\section{Implications}

We did not find that outcomes for the gravid rat held for the human female. In particular we did not find significant cognitive improvements in functioning in pregnant women or in mothers relative to controls as have been found in earlier animal work. In this context it is important to acknowledge that human and animal studies do differ substantially, in that domains of cognition are different (for example water mazes in comparison to verbal recall), and that improvements in spatial functioning in animals are not universally found, with some studies finding deficits in rats during the third trimester. ${ }^{32}$ Nonetheless, there were no indications of improvement in our study. Moreover, although cognitive tests will differ for humans and rats, tests are similar in that they reflect hippocampal functioning (see for example Lye et $a l)^{34}$ so some degree of consistency might have been expected. Whether the differences between humans and animals are a result of differing biological substrates, the social environment or their interplay remains to be explored. Perhaps, more importantly from the perspective of mothers to be and those caring for them, we were also not able to establish substantial or consistent cognitive deficits. Except in a brief period in later pregnancy, these findings challenge the common myth that women develop 'placenta brain' or 'baby brain'. We found no deficits on memory tests in particular. Since both women and 
their partners believe that women experience cognitive deficits in pregnancy (see Christensen et al), ${ }^{11}$ women and their partners need to be encouraged to be less automatic in their willingness to attribute common memory lapses to the salient causal factor of a growing or new baby. Obstetricians, general family doctors and midwives may need to use the findings from this study to promote the view that 'placenta brain' is not inevitable, and that perceptions of impairment may reflect emotional or other unknown factors. Not so long ago pregnancy was 'confinement' and motherhood meant the end of career aspirations. Our results challenge the view that mothers are anything other than the intellectual peers of their contemporaries.

Helen Christensen, BA, BPsychol, PhD, Liana S. Leach, BAppPych, PhD, Centre for Mental Health Research, The Australian National University, Canberra; Andrew Mackinnon, BSC, PhD, ORYGEN Research Centre, The University of Melbourne, Australia

Correspondence: Helen Christensen, Centre for Mental Health Research, The Australian National University, Canberra ACT 0200, Australia. Email: Helen.Christensen@anu.edu.au

First received 18 May 2009, final revision 8 Oct 2009, accepted 23 Oct 2009

\section{Funding}

The PATH Through Life Project is funded by NHMRC Grant number 418039. H.C. is funded by NHMRC Fellowship number 525411 and L.L. by NHMRC PhD Scholarship number by NHMRC

\section{Acknowledgements}

The authors would like to thank K. J. Anstey, A. F. Jorm, P. J. Butterworth P. A. Jacomb and K. Maxwell for contributions to the design of the PATH Through Life Project. Amelia Gulliver assisted in developing the reference list.

\section{References}

1 Crystal G. What is Pregnancy Brain? Wise Geek (http://www.wisegeek.com/ what-is-pregnancy-brain.htm)

2 BabyCenter Medical Advisory Board. Forgetfulness During Pregnancy. BabyCenter (http://www.babycenter.com/0_forgetfulness-duringpregnancy_236.bc).

3 Denis Campbell. Pregnancy 'does cause memory loss'. The Guardian 2008; 3 February (http://www.guardian.co.uk/science/2008/feb/03/ medicalresearch.pregnancy).

4 Henry JD, Rendell PJ. A review of the impact of pregnancy on memory function. J Clin Exp Neuropsychol 2007; 29: 793-803.

5 Brindle PM, Brown MW, Brown J, Griffith HB, Turner GM. Objective and subjective memory impairment in pregnancy. Psychol Med 1991; 21: 647-53.

6 de Groot R, Hornstra R, Roozendaal N, Jolles J (2003) Memory performance, but not information processing speed, may be reduced during early pregnancy. J Clin Exp Neuropsychol 2003; 25: 482-8.

7 Janes $C$, Casey P, Huntsdale C, Angus G. Memory in pregnancy. I: Subjective experiences and objective assessment of implicit, explicit and working memory in primigravid and primiparous women. J Psychosom obstet Gynaecol 1999; 20: 80-7.

8 Rendell PG, Henry JD (2008) Prospective-memory functioning is affected during pregnancy and postpartum. J Clin Exp Neuropsychol 2008; 30: 913-9.
9 Sharp K, Brindle PM, Brown MW, Turner GM. Memory loss during pregnancy. Br J Obstet Gynaecol 1993; 100: 209-15.

10 Shetty DN, Pathak SS. Correlation between plasma neurotransmitters and memory loss in pregnancy. J Reprod Med 2002; 47: 494-6.

11 Christensen $\mathrm{H}$, Poyser C, Pollit P, Cubis J. Pregnancy may confer a selective cognitive advantage. J Reprod Infant Psychol 1999; 17: 7-25.

12 Condon J, Derham D, Kneebone A. Cognitive functioning during pregnancy: a controlled investigation using psychometric testing. Int J Prenat Perinat Stud 1999; 199: 212

13 de Groot R, Vuurman E, Hornstra G, Jolles J. Differences in cognitive performance during pregnancy and early motherhood. Psychol Med 2006; 36: 1023-32.

14 Jarrahi-Zadeh A, Kane FJ, Van de Castlf RL, Lachenbruch PA, Ewing JA Emotional and cognitive changes in pregnancy and early puerperium. $\mathrm{Br} J$ Psychiatry 1969; 115: 797-805.

15 Keenan PA, Yaldoo DT, Stress ME, Fuerst DR, Ginsburg KA. Explicit memory in pregnant women. Am J Obstet Gynecol 1998; 179: 731-7.

16 Silber M, Almkvist O, Larsson B, Uvnas-Moberg K. Temporary peripartal impairment in memory and attention and its possible relation to oxytocin concentration. Life Sci 1990; 47: 57-65.

17 Crawley RA, Dennison $\mathrm{K}$, Carter C. Cognition in pregnancy and the first year post-partum. Psychol Psychother 2003; 76: 69-84.

18 Harris N, Deary I, Harris M, Lees M, Wilson J. Peripartal cognitive impairment: secondary to depression? Br J Health Psychol 1996; 1: 127-36.

19 Swain AM, O'Hara MW, Starr KR, Gorman LL. A prospective study of sleep mood, and cognitive function in postpartum and nonpostpartum women. Obstet Gynecol 1997; 90: 381-6.

20 Casey P. A longitudinal study of cognitive performance during pregnancy and new motherhood. Arch Womens Ment Health 2000; 3: 65-76.

21 Kinsley $\mathrm{CH}$, Madonia L, Gifford GW, Tureski K, Griffin GR, Lowry C, et al. Motherhood improves learning and memory. Nature 1999; 402: 137-8.

22 Bodensteiner KJ, Cain P, Ray AS, Hamula LA. Effects of pregnancy on spatial cognition in female Hooded Long-Evans rats. Horm Behav 2006; 49: 303-14.

23 Kinsley $\mathrm{CH}$, Bardi M, Karelina K, Rima B, Christon L, Friedenberg J, et al. Motherhood induces and maintains behavioral and neural plasticity across the lifespan in the rat. Arch Sex Behav 2008; 37: 43-56.

24 Love G, Torrey N, McNamara I, Morgan M, Banks M, Hester NW, et al. Maternal experience produces long-lasting behavioral modifications in the rat. Behav Neurosci 2005; 119: 1084-96.

25 Kinsley CH, Lambert KG. The maternal brain. Sci Am 2006; 294: 72-9.

26 Jorm AF, Korten AE, Rodgers B, Jacomb PA, Christensen H. Sexual orientation and mental health: results from a community survey of young and middle-aged adults. Br J Psychiatry 2002; 180: 423-7.

27 Wechsler D. Manual for the Wechsler Adult Intelligence Scale - Revised (WAIS-R). Psychological Corporation, 1981.

28 Smith A. Symbol-Digit Modalities Test (SDMT) Manual. Western Psychological Services, 1982.

29 Wechsler D. A standardized memory scale for clinical use. J Psychol 1945; 19: 87-95.

30 Delis DC, Kramer JH, Kaplan E, Ober BA. California Verbal Learning Test. Psychological Corporation/Harcourt Brace Jovanovich, 1987.

31 Goldberg D, Bridges K, Duncan-Jones P, Grayson D. Detecting anxiety and depression in general medical settings. Br Med J 1988; 297: 897-9.

32 Brown H, Prescott R. Applied Mixed Models in Medicine. Wiley, 2006.

33 Galea LAM, Ormerod BK, Sampath XK, Wikie DM, Phelps MT. Spatial working memory and hippocampal size across pregnancy in rats. Horm Behav 2000; 37: 86-95.

34 Lye TC, Piguet O, Grayson DA, Creasey H, Ridley L, Bennett HP, et al. Hippocampal size and memory functioning in the ninth and tenth decades of life: the Sydney Older Person's Study. J Neurol Neurosurg Psychiatry 2004; 75: $548-54$ 